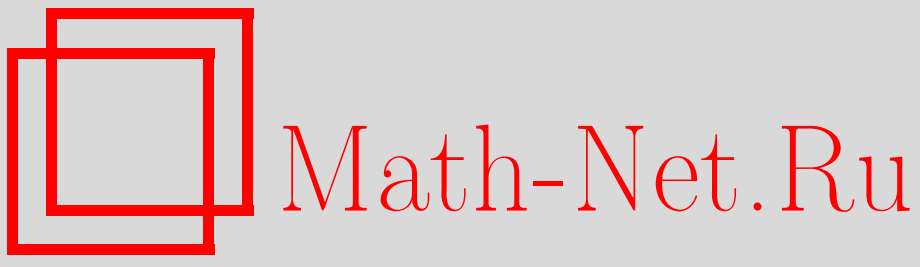

В. И. Питербарг, Ю. Н. Тюрин, Многомерные ранговые корреляции: гауссовское поле на прямом произведении сфер, Теория вероятн. и ее примен., 2000, том 45, выпуск 2, 236250

DOI: https://doi.org/10.4213/tvp461

Использование Общероссийского математического портала Math-Net.Ru подразумевает, что вы прочитали и согласны с пользовательским соглашением http://www.mathnet.ru/rus/agreement

Параметры загрузки:

IP: 54.80 .73 .141

26 апреля 2023 г., $17: 23: 41$

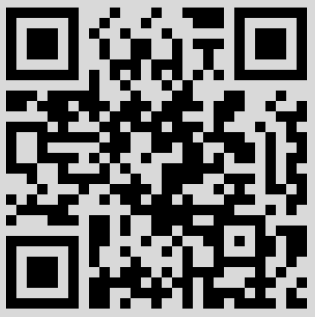


(C) $2000 \mathrm{r}$.

\author{
ПИТЕРБАРГ В.И., ТЮРИН Ю.Н.*
}

\title{
МНОГОМЕРНЫЕ РАНГОВЫЕ КОРРЕЛЯЩИИ: ГАУССОВСКОЕ ПОЛЕ НА ПРЯМОМ ПРОИЗВЕДЕНИИ СФЕР ${ }^{1)}$
}

\begin{abstract}
Предложен асимптотический метод для проверки независимости компонент случайного вектора. Правило проверки основано на ранжировании линейных комбинаций координат наблюдений и последующем применении принципа Роя «объединения пересечений».
\end{abstract}

Ключевые слова и фразы: многомерная выборка, ранги, тау Кендалла, слабая сходимость.

1. Введение. Одна из базисных проблем статистики, которая встречается во многих приложениях, касается зависимости и независимости двух групп случайных переменных. Для случайных величин и случайных векторов, совместное распределение которых нормально, проверка независимости сводится к проверке гипотез о равенстве нулю коэффициентов корреляции. (См., например, $[11$, гл. 29], [12, гл. 4 и 9].) Для пары одномерных случайных величин с произвольными (но непрерывными) распределениями для проверки независимости по выборке тоже можно использовать коэффициенты корреляции, но уже ранговые, основанные на рангах наблюдений. Когда признаки независимы, вычисленные по выборке коэффициенты ранговой корреляции распределены свободно, их законы распределений известны, а потому гипотезу о независимости (о равенстве нулю их ожидаемых значений) можно проверить. (См., например, $[13$, гл. 3, п. 6], $[14$, гл. 8].) $\mathrm{K}$ сожалению, для векторных наблюдений неизвестно такое обобщение операции ранжирования, которое бы приводило к свободным от распределения статистическим правилам, хотя сама проблема многомерного ранжирования давно привлекает к себе внимание. (См., например, [8], [15], [16].)

\footnotetext{
${ }^{*}$ Московский государственный университет им. М. В. Ломоносова, механикоматематический факультет, Воробьевы горы, 119899 Москва, Россия; е-таil: piter@mech.math.msu.su

1) Работа выполнена при поддержке Российского фонда фундаментальных исследованшй (первый автор - проекты 97-01-00648, 98-01-00524; второй автор - проекты 96-01-00336, 97-01-00648).
} 
В этой статье мы предлагаем обобщение коэффициента ранговой корреляции Кендалла для векторных выборок. Оно основано на применении направленного ранжирования, которое мы ввели и использовали ранее, [7], вместе с принципом включения-исключения Роя, [9], [10]. По методу Роя критическое множество составляется как объединение критических множеств для проверки тех простых гипотез, из которых состоит проверяемая гипотеза. Гипотезу о независимости двух случайных векторов мы представляем в виде пересечения гипотез о независимости всех линейных форм от этих векторов.

Более точно, пусть $\mathbf{X}$ и $\mathbf{Y}$ суть $p$ - и $q$-мерные случайные векторы и

$$
\left(\mathbf{X}_{1}, \mathbf{Y}_{1}\right),\left(\mathbf{X}_{2}, \mathbf{Y}_{2}\right), \ldots,\left(\mathbf{X}_{n}, \mathbf{Y}_{n}\right)
$$

представляют собой $n$ независимых реализаций $(\mathbf{X}, \mathbf{Y})$. Гипотеза

$$
H \text { : случайные векторы } \mathbf{X} \text { и } \mathbf{Y} \text { независимы }
$$

влечет за собой гипотезы $H_{\alpha, \beta}$ о том, что независимы одномерные случайные величины $\alpha^{\top} \mathbf{X}$ и $\beta^{\top} \mathbf{Y}$, где $\alpha \in \mathbf{R}^{p}$ и $\beta \in \mathbf{R}^{q}$ суть ненулевые неслучайные векторы. (Здесь и далее все векторы - это векторыстолбцы.) Следуя [9], гипотеза $H$ отвергается, если отвергается хотя бы одна из гипотез $H_{\alpha, \beta}$.

Гипотезу $H_{\alpha, \beta}$ можно проверять многими различными статистическими критериями. Среди таких критериев коэффициент ранговой корреляции Кендалла показывает, насколько согласованно возрастают или убывают две случайные последовательности. По определению,

$$
\tau=\tau_{n}(\alpha, \beta)=\frac{1}{n(n-1)} \sum_{1 \leqslant i \neq j \leqslant n} s\left[\left(\alpha^{\top} \mathbf{X}_{i}-\alpha^{\top} \mathbf{X}_{j}\right)\left(\beta^{\top} \mathbf{Y}_{i}-\beta^{\top} \mathbf{Y}_{j}\right)\right]
$$

где $s[x]$ есть знак $x$,

$$
s[x]=\left\{\begin{array}{rrr}
1, & \text { если } x>0 \\
-1, & \text { если } x<0 \\
0, & \text { если } & x=0
\end{array}\right.
$$

По Кендаллу, [3], $H_{\alpha, \beta}$ следует отвергнуть, если $\left|\tau_{n}(\alpha, \beta)\right|$ слишком далеко отступает от нуля. В качестве критериальной статистики для проверки $H$ мы предлагаем использовать далее

$$
\sup _{\alpha, \beta}\left|\tau_{n}(\alpha, \beta)\right|
$$

и отвергать $H$, когда (4) превосходит критический уровень. Подобно коэффициенту ранговой корреляции Кендалла, максимальное значение (4) равно 1. Это максимальное значение достигается, если при каких-то $\alpha \in \mathbf{R}^{p}, \beta \in \mathbf{R}^{q}$ полностью совпадают ранги последовательностей $\alpha^{\top} \mathbf{X}_{1}, \ldots, \alpha^{\top} \mathbf{X}_{n}$ и $\beta^{\top} \mathbf{Y}_{1}, \ldots, \beta^{\top} \mathbf{Y}_{n} . \mathrm{K}$ сожалению, статистика (4) 
не является распределенной свободно даже при справедливости гипотезы (2): ее распределение зависит от распределения случайных наблюдений (1), так же, как и распределение случайного поля (3). Возможны, однако, асимптотические (при $n \rightarrow \infty$ ) применения критерия, основанного на статистике (4): как будет показано ниже, для этого достаточно оценить по выборке всего один числовой параметр.

Перейдем к асимптотическим свойствам статистики (4) и рассмотрим стандартизованный коэффициент ранговой корреляции:

$$
T_{n}(\alpha, \beta):=\sigma_{n}^{-1} \sum_{1 \leqslant i \neq j \leqslant n} s\left[\left(\alpha^{\top} \mathbf{X}_{i}-\alpha^{\top} \mathbf{X}_{j}\right)\left(\beta^{\top} \mathbf{Y}_{i}-\beta^{\top} \mathbf{Y}_{j}\right)\right]
$$

где

$$
\sigma_{n}^{2}=\frac{2}{9} n(n-1)(2 n+5)
$$

- это дисперсия случайной величины $n(n-1) \tau_{n}(\alpha, \beta)$ при выполнении гипотезы (2).

Отметим, что, подобно $\tau_{n}(\alpha, \beta)$, статистика $T_{n}(\alpha, \beta)$ постоянна вдоль любого «двумерного направления», так что для любых $\alpha, \beta$ и произвольного $t>0$ :

$$
T_{n}(t \alpha, \beta)=T_{n}(\alpha, t \beta)=T_{n}(\alpha, \beta)
$$

Kроме тоro,

$$
T_{n}(-\alpha, \beta)=T_{n}(\alpha,-\beta)=-T_{n}(\alpha, \beta)
$$

Поэтому

$$
\sup _{\alpha, \beta}\left|T_{n}(\alpha, \beta)\right|=\sup _{|\alpha|=|\beta|=1} T_{n}(\alpha, \beta) .
$$

Вместо прямого произведения единичных сфер в правой части (8) можно взять любое множество, которое пересекается с каждой четвертьплоскостью $\{(t \alpha, s \beta), t \geqslant 0, s \geqslant 0\}$. В частности, при доказательстве теоремы 1 вместо прямого произведения сфер мы рассмотрим прямое произведение единичных кубов.

В теореме 1 мы доказываем, что при гипотезе $H$ и некоторых условиях регулярности, касающихся плотностей векторов $\mathbf{X}$ и $\mathbf{Y}$, статистика (8) при $n \rightarrow \infty$ сходится по распределению $\mathrm{x} \sup _{|\alpha|=|\beta|=1} Z(\alpha, \beta)$, где $Z(\alpha, \beta), \alpha \in \mathbf{R}^{p}, \beta \in \mathbf{R}^{q},-$ некоторое гауссовское поле.

Теорема 2 дает асимптотическую формулу для вычисления вероятности

$$
\mathbf{P}\left\{\sup _{|\alpha|=|\beta|=1} Z(\alpha, \beta)>z\right\},
$$

когда $z$ неограниченно возрастает. Следовательно, для больших $n$ и малых уровней значимости мы отвергаем гипотезу $H$ на уровне $\varepsilon>0$, если

$$
\sup _{|\alpha|=|\beta|=1} T_{n}(\alpha, \beta)>z_{\varepsilon}
$$


где $z_{\varepsilon}$ следует вычислять по асимптотической формуле из теоремы 2. Приближение для больших выборок для верхних критических значений статистики (8), которое дают теоремы 1 и 2, зависит от распределения выборок только через постоянный множитель $K_{\mathbf{X}} K_{\mathbf{Y}}$, см. (14) в п. 2. Эту постоянную приходится оценивать по имеющимся выборкам. Состоятельная оценка для $K_{\mathbf{X}} K_{\mathbf{Y}}$ дана в п. 5. Указанное приближение может быть использовано для проверки $H$ с малыми уровнями значимости. К сожалению, трудно оценить теоретически, какова (относительная) ошибка предложенной двустадийной аппроксимации. Мы надеемся, что это можно будет сделать численно. Тогда станет более ясным, насколько практически полезными окажутся изложенные результаты. ${ }^{2)}$

2. Основные результаты. Первый из них относится к слабой сходимости $T_{n}$ к гауссовскому полю. Хотя траектории $T_{n}$ не являются непрерывными, будет установлена слабая сходимость в пространстве функций на $S^{p-1} \times S^{q-1}$, не имеющих разрывов второго рода, снабженном равномерной метрикой.

Теорема 1. Пусть $\mathbf{X}_{1}, \ldots, \mathbf{X}_{n} u \mathbf{Y}_{1}, \ldots, \mathbf{Y}_{n}$ суть $p$ - и q-мерные независимые выборки из распределении, имеюиих непрерывные плотносmи $p_{\mathbf{X}}$ и $p_{\mathbf{Y}}$ относительно лебеговской меры в $\mathbf{R}^{p} u \mathbf{R}^{q}$ соответственно. Предположим, что для некоторых положительных постоянных $C, \gamma$ и для всех $\mathbf{x} \in \mathbf{R}^{p}, \mathbf{y} \in \mathbf{R}^{q}$

$$
p_{\mathbf{X}}(\mathbf{x}) \leqslant \frac{C}{1+|\mathbf{x}|^{p+\gamma}}, \quad p_{\mathbf{Y}}(\mathbf{y}) \leqslant \frac{C}{1+|\mathbf{y}|^{q+\gamma}} .
$$

Пусть $Z(\alpha, \beta), \alpha \in \mathbf{R}^{p}, \beta \in \mathbf{R}^{q}, \alpha \neq \mathbf{0}, \beta \neq \mathbf{0}$, обозначает случайное гауссовское поле такое, ито

$$
\begin{gathered}
\mathbf{E} Z(\alpha, \beta)=0 \\
r\left(\alpha, \beta ; \alpha^{\prime}, \beta^{\prime}\right)=\mathbf{E} Z(\alpha, \beta) Z\left(\alpha^{\prime}, \beta^{\prime}\right)=r_{\mathbf{X}}\left(\alpha, \alpha^{\prime}\right) r_{\mathbf{Y}}\left(\beta, \beta^{\prime}\right),
\end{gathered}
$$

$2 \partial e$

$$
\begin{aligned}
& r_{\mathbf{X}}\left(\alpha, \alpha^{\prime}\right)=3 \mathbf{E} s\left[\alpha^{\top}\left(\mathbf{X}_{1}-\mathbf{X}_{3}\right) \alpha^{\prime \top}\left(\mathbf{X}_{2}-\mathbf{X}_{3}\right)\right] \\
& r_{\mathbf{Y}}\left(\beta, \beta^{\prime}\right)=3 \mathbf{E} s\left[\beta^{\top}\left(\mathbf{Y}_{1}-\mathbf{Y}_{3}\right) \beta^{\prime \top}\left(\mathbf{Y}_{2}-\mathbf{Y}_{3}\right)\right]
\end{aligned}
$$

Тогда $T_{n}(\alpha, \beta) \Rightarrow Z(\alpha, \beta) n p u n \rightarrow \infty$ слабо в $D\left(S^{p-1} \times S^{q-1}\right)$ в равномерной метрике, и потому по распределению

$$
\sup _{|\alpha|=|\beta|=1} T_{n}(\alpha, \beta) \Longrightarrow \sup _{|\alpha|=|\beta|=1} Z(\alpha, \beta) \quad n p u n \rightarrow \infty
$$

2) Т.А. Романова недавно провела такие расчеты методом Монте-Карло для $p=$ $q=2, n=100 \div 500$ и нескольких разлипых распределений. Асимптотические результаты этой статьи оказались с ними в хорошем согласии. - Примечаяие при корректype. 
Доказательство теоремы 1 вынесено в п. 3. Чтобы сформулировать следующую теорему, нам потребуется некоторая числовая характеристика многомерного распределения. Для ненулевых $\alpha \in \mathbf{R}^{p}, \beta \in \mathbf{R}^{q}$ oпределим матрицы

$$
\begin{array}{r}
B_{\mathbf{X}}(\alpha)=12 \int_{\mathbf{R}^{p}} d \mathbf{x} \int_{\alpha^{\top} \mathbf{y}=0} \mathbf{y} d \mathbf{y} \int_{\alpha^{\top} \mathbf{z}=0} \mathbf{z}^{\top} d \mathbf{z} p_{\mathbf{X}}(\mathbf{x}-\mathbf{y}) \\
\times p_{\mathbf{X}}(\mathbf{x}-\mathbf{z}) p_{\mathbf{X}}(\mathbf{x}), \\
B_{\mathbf{Y}}(\beta)=12 \int_{\mathbf{R}^{q}} d \mathbf{x} \int_{\beta^{\top} \mathbf{y}=0} \mathbf{y} d \mathbf{y} \int_{\beta^{\top} \mathbf{z}=0} \mathbf{z}^{\top} d \mathbf{z} p_{\mathbf{Y}}(\mathbf{x}-\mathbf{y}) \\
\times p_{\mathbf{Y}}(\mathbf{x}-\mathbf{z}) p_{\mathbf{Y}}(\mathbf{x}) .
\end{array}
$$

В формулах (12) и (13), а также последующих, им подобных, $\int_{\alpha^{\top} \mathbf{y}=0} f(\mathbf{y}) d \mathbf{y}$ означает интегрирование по мере Лебега на подпространстве $\alpha^{\top} \mathbf{y}=0$. Для интегрируемой функции $f(\cdot)$ такие интегралы существуют для почти всех $\alpha$. Для матрицы $B$ размера $d \times d$ обозначим через $\delta(B)$ сумму ее главных миноров порядка $d-1$. Далее, определим постоянные

$$
K_{\mathbf{X}}=\int_{S^{p-1}} \sqrt{\delta\left(B_{\mathbf{X}}(\alpha)\right)} S(d \alpha), \quad K_{\mathbf{Y}}=\int_{S^{q-1}} \sqrt{\delta\left(B_{\mathbf{Y}}(\beta)\right)} S(d \beta),
$$

где интегрирование производится по единичныг сферам, а $S(d \alpha), S(d \beta)$ обозначают элементы поверхностей $S^{p-1}$ и $S^{q-1}$ соответственно.

Теорема 2. Для гауссовского поля $Z(\alpha, \beta)$, вөеденного в теоремe 1,

$$
\mathbf{P}\left\{\sup _{|\alpha|=|\beta|=1} Z(\alpha, \beta)>z\right\}=\frac{K_{\mathbf{X}} K_{\mathbf{Y}}}{2(\sqrt{2 \pi})^{p+q-1}} z^{p+q-3} e^{-z^{2} / 2}(1+o(1)),
$$

когда $z \rightarrow \infty$.

Сформулируем результаты статьи в виде правила для проверки независимости двух многомерных признаков $\mathbf{X}$ и $\mathbf{Y}$ по выборке (1) большого объема.

1. По выборке (1) вычисляем статистику

$$
T_{n}:=\sup _{\alpha \in S^{p-1}, \beta \in S^{q-1}} T_{n}(\alpha, \beta),
$$

где $T_{n}(\alpha, \beta)$ задано формулами (5) и (6). (Приближенное вычисление максимального значения $T_{n}(\alpha, \beta)$ можно осуществлять методом МонтеКарло, случайно выбирая точки $\alpha \in S^{p-1}$ и $\beta \in S^{q-1}$.)

2. По формулам (43) из п. 5 вычисляем $K_{\mathbf{X}}^{n}$ й $K_{\mathbf{Y}}^{n}$.

3. Выбрав уровень значимости $\varepsilon>0$, решаем уравнение

$$
\frac{K_{\mathbf{X}}^{n} K_{\mathbf{Y}}^{n}}{2(\sqrt{2 \pi})^{p+q-1}} z^{p+q-2} e^{-z^{2} / 2}=\varepsilon
$$

и находим $z=z_{\varepsilon}(n)$.

4. Отвергаем гипотезу независимости (2) на (приближенном) уровне $\varepsilon$, если $T_{n}>z_{\varepsilon}(n)$. 
3. Доказательство теоремы 1. Введем случайные величины

$$
\begin{aligned}
& A\left(\alpha, \mathbf{X}_{j}\right)=\mathbf{E}\left\{s\left[\alpha^{\top}\left(\mathbf{X}_{1}-\mathbf{X}_{j}\right)\right] \mid \mathbf{X}_{j}\right\} \\
& B\left(\beta, \mathbf{Y}_{j}\right)=\mathbf{E}\left\{s\left[\beta^{\top}\left(\mathbf{Y}_{1}-\mathbf{Y}_{j}\right)\right] \mid \mathbf{Y}_{j}\right\}
\end{aligned}
$$

Заметим, что при гипотезе $H$

$$
\mathbf{E}\left\{s\left[\alpha^{\top}\left(\mathbf{X}_{1}-\mathbf{X}_{j}\right) \beta^{\top}\left(\mathbf{Y}_{1}-\mathbf{Y}_{j}\right)\right] \mid\left(\mathbf{X}_{j}, \mathbf{Y}_{j}\right)\right\}=A\left(\alpha, \mathbf{X}_{j}\right) B\left(\beta, \mathbf{Y}_{j}\right)
$$

Имея это в виду, представим $T_{n}(\alpha, \beta)$ в виде:

$$
T_{n}(\alpha, \beta)=2(n-1) \sigma_{n}^{-1} \sum_{i=1}^{n} A\left(\alpha, \mathbf{X}_{i}\right) B\left(\beta, \mathbf{Y}_{i}\right)+\Delta_{n}(\alpha, \beta)
$$

где

$$
\begin{aligned}
\Delta_{n}(\alpha, \beta)=\sigma_{n}^{-1} \sum_{1 \leqslant i \neq j \leqslant n}(s & {\left[\alpha^{\top}\left(\mathbf{X}_{i}-\mathbf{X}_{j}\right) \beta^{\top}\left(\mathbf{Y}_{i}-\mathbf{Y}_{j}\right)\right] } \\
& \left.-A\left(\alpha, \mathbf{X}_{i}\right) B\left(\beta, \mathbf{Y}_{i}\right)-A\left(\alpha, \mathbf{X}_{j}\right) B\left(\beta, \mathbf{Y}_{j}\right)\right) .
\end{aligned}
$$

Далее мы покажем, что:

(i) $\sup _{\alpha, \beta}\left|\Delta_{n}(\alpha, \beta)\right| \longrightarrow 0$ по вероятности, когда $n \rightarrow \infty$;

(ii) $2(n-1) \sigma_{n}^{-1} \sum_{i=1}^{n} A\left(\alpha, \mathbf{X}_{i}\right) B\left(\beta, \mathbf{Y}_{i}\right) \Longrightarrow Z(\alpha, \beta)$ при $n \rightarrow \infty$ слабо в $C\left(S^{p-1} \times S^{q-1}\right)$.

Эти два утверждения составляют доказательство теоремы 1.

Доказ ательст во утверждения (i). Отметим, что, подобно $T_{n}, \Delta_{n}(\alpha, \beta)$ постоянна вдоль каждой четверть-плоскости $\{(t \alpha, s \beta), t \geqslant 0, s \geqslant 0\}$. Можно поэтому считать, что $\alpha$ пробегает поверхность единичного куба в $\mathbf{R}^{p}$ с центром в нуле, а $\beta$ пробегает поверхность такого же куба в $\mathbf{R}^{q}$. Рассмотрим разбиение поверхностей этих кубов на, соответственно, $(p-1)$ - и $(q-1)$-мерные кубики, длина ребра которых $h=h(n)$ далее будет уточнена. Пусть $\Lambda=\Lambda(n)$ и $M=M(n)$ обозначают совокупности центров этих кубиков в пространствах $\mathbf{R}^{p}$ и $\mathbf{R}^{q}$ соответственно. Кубики с центрами $a \in \Lambda, b \in M$ обозначим через $U(a)$ и $V(b)$ соответственно. Заметим, что, поскольку $\sup \left|\Delta_{n}(\alpha, \beta)\right|=\sup \Delta_{n}(\alpha, \beta)$, можно изучать $\Delta_{n}$ вместо $\left|\Delta_{n}\right|$. Поскольку

$$
\sup _{\alpha, \beta} \Delta_{n}(\alpha, \beta)=\max _{a \in \Lambda, b \in M} \sup _{\alpha \in U(a), \beta \in V(b)} \Delta_{n}(\alpha, \beta),
$$

наша задача теперь - доказать, что

$$
\mathbf{P}\left\{\max _{a \in \Lambda, b \in M} \sup _{\alpha \in U(a), \beta \in V(b)} \Delta_{n}(\alpha, \beta)>\varepsilon\right\} \rightarrow 0
$$


при $n \rightarrow \infty$. Очевидно, что

$$
\begin{aligned}
& \mathbf{P}\left\{\max _{a \in \Lambda, b \in M} \sup _{\alpha \in U(a), \beta \in V(b)} \Delta_{n}(\alpha, \beta)>\varepsilon\right\} \\
& \quad \leqslant \sum_{a \in \Lambda} \sum_{b \in M} \mathbf{P}\left\{\sup _{\alpha \in U(a), \beta \in V(b)} \Delta_{n}(\alpha, \beta)>\varepsilon\right\} .
\end{aligned}
$$

Число слагаемых в правой части (17) пропорционально $h^{-(p+q-2)}$. Наша ближайшая цель состоит в том, чтобы оценить сверху общий член (17) так, чтобы при подходящем выборе $h=h(n)$ оценка суммы в (17) стремилась к нулю при $n \rightarrow \infty$. Заметим, что

$$
\sup _{\alpha \in U(a), \beta \in V(b)} \Delta_{n}(\alpha, \beta) \leqslant \Omega_{1}+\Omega_{2}+\Omega_{3}
$$

где

$$
\begin{aligned}
& \Omega_{1}=\sigma_{n}^{-1} \sup _{\alpha \in U(a), \beta \in V(b)} \mid \sum_{1 \leqslant i \neq j \leqslant n}\left(s\left[\alpha^{\top}\left(\mathbf{X}_{i}-\mathbf{X}_{j}\right) \beta^{\top}\left(\mathbf{Y}_{i}-\mathbf{Y}_{j}\right)\right]\right. \\
& \left.-s\left[a^{\top}\left(\mathbf{X}_{i}-\mathbf{X}_{j}\right) b^{\top}\left(\mathbf{Y}_{i}-\mathbf{Y}_{j}\right)\right]\right) \mid \\
& \Omega_{2}=\sigma_{n}^{-1} \mid \sum_{1 \leqslant i \neq j \leqslant n}\left(s\left[a^{\top}\left(\mathbf{X}_{i}-\mathbf{X}_{j}\right) b^{\top}\left(\mathbf{Y}_{i}-\mathbf{Y}_{j}\right)\right]\right. \\
& \left.-A\left(a, \mathbf{X}_{i}\right) B\left(b, \mathbf{Y}_{i}\right)-A\left(a, \mathbf{X}_{j}\right) B\left(b, \mathbf{Y}_{j}\right)\right) \\
& \Omega_{3}=2(n-1) \sigma_{n}^{-1} \mid \sup _{\alpha \in U(a), \beta \in V(b)} \sum_{i=1}^{n}\left(A\left(\alpha, \mathbf{X}_{i}\right) B\left(\beta, \mathbf{Y}_{i}\right)\right. \\
& \left.-A\left(a, \mathbf{X}_{i}\right) B\left(b, \mathbf{Y}_{i}\right)\right)
\end{aligned}
$$

По неравенству Чебышева

$$
\begin{aligned}
& \mathbf{P}\left\{\sup _{\alpha \in U(a), \beta \in V(b)} \Delta_{n}(\alpha, \beta)>\varepsilon\right\} \\
& \quad \leqslant \mathbf{P}\left\{\Omega_{1} \geqslant \frac{\varepsilon}{3}\right\}+\mathbf{P}\left\{\Omega_{2} \geqslant \frac{\varepsilon}{3}\right\}+\mathbf{P}\left\{\Omega_{3} \geqslant \frac{\varepsilon}{3}\right\} \leqslant\left(\frac{\varepsilon}{3}\right)^{-2 d} \sum_{k=1}^{3} \mathbf{E} \Omega_{k}^{2 d}
\end{aligned}
$$

Надо так оценить $\mathbf{E} \Omega_{k}^{2 d}$ сверху, и с таким значением $d$, чтобы

$$
h^{-(p+q-2)} \mathbf{E} \Omega_{k}^{2 d} \rightarrow 0
$$

для всех $k=1,2,3$, когда $n \rightarrow \infty$. и (16).

Сначала мы установим некоторые полезные свойства функций (15) 
Лемма. В предположениях теоремы 1 для некоторой неслучайной константы $C$ и для всех $\alpha, \beta, \alpha^{\prime}, \beta^{\prime}$

$$
\begin{aligned}
& \left|A\left(\alpha^{\prime}, \mathbf{X}\right)-A\left(\alpha^{\prime \prime}, \mathbf{X}\right)\right| \leqslant C\left|\alpha^{\prime}-\alpha^{\prime \prime}\right| \\
& \left|B\left(\beta^{\prime}, \mathbf{Y}\right)-B\left(\beta^{\prime \prime}, \mathbf{Y}\right)\right| \leqslant C\left|\beta^{\prime}-\beta^{\prime \prime}\right|
\end{aligned}
$$

Д ок аз а т л в с т в о. Достаточно доказать первое неравенство. Доказательство второго получается из него изменением обозначений. Рассмотрим условное математическое ожидание

$$
A(\alpha, \mathbf{x})=\mathbf{E}\left\{s\left[\alpha^{\top}\left(\mathbf{X}_{1}-\mathbf{X}_{2}\right)\right] \mid \mathbf{X}_{2}=\mathbf{x}\right\}
$$

Очевидно, что

$$
A\left(\alpha^{\prime}, \mathbf{x}\right)=1-2 \mathbf{P}\left\{\alpha^{\prime \top} \mathbf{X}_{1}<\alpha^{\prime \top} \mathbf{x}\right\}
$$

Рассмотрим ортогональное разложение $\alpha^{\prime}=\alpha^{\prime \prime}+\delta \nu, \alpha^{\prime}, \alpha^{\prime \prime}, \nu \in \mathbf{R}^{p}$, $\delta \in \mathbf{R}^{1}$, где $\nu \perp \alpha^{\prime \prime},\left|\alpha^{\prime \prime}\right|=|\nu|=1$. Имеем

$$
\mathbf{P}\left\{\alpha^{\prime \top} \mathbf{X}<\alpha^{\prime \top} \mathbf{x}\right\}=\mathbf{P}\left\{\alpha^{\prime \prime \top} \mathbf{X}<\alpha^{\prime \prime \top} \mathbf{x}\right\}+f(\delta, \mathbf{x}),
$$

где

$$
f(\delta, \mathbf{x})=\int_{\alpha^{\prime \prime \top} \mathbf{z}=0} d \mathbf{z} \int_{0}^{\delta \nu^{\top} \mathbf{z}} p_{\mathbf{X}}\left(\mathbf{x}+\mathbf{z}+\mathbf{y} \alpha^{\prime \prime}\right) d \mathbf{y}
$$

Из предположения (10) следует, что функция $f(\delta, \mathbf{x})$ непрерывно дифференцируема по $\delta$ и что для достаточно малых $\delta$ ее производная

$$
f^{\prime}(\delta, \mathbf{x}):=\frac{\partial}{\partial \delta} f(\delta, \mathbf{x})=-\int_{\alpha^{\prime \prime} \top \mathbf{z}=0}\left(\nu^{\top} \mathbf{z}\right) p_{\mathbf{X}}\left(\mathbf{x}+\mathbf{z}-\delta \nu^{\top} \mathbf{z} \alpha^{\prime \prime}\right) d \mathbf{z}
$$

равномерно ограничена по $\mathbf{x}, \alpha^{\prime \prime}$ и $\nu$. Следовательно, для некоторого $C$

$$
\left|\mathbf{P}\left\{\alpha^{\prime \top} \mathbf{X}<\alpha^{\prime \top} \mathbf{x}\right\}-\mathbf{P}\left\{\alpha^{\prime \prime \top} \mathbf{X}<\alpha^{\prime \prime \top} \mathbf{x}\right\}\right| \leqslant C\left|\alpha^{\prime}-\alpha^{\prime \prime}\right|
$$

что доказывает утверждение леммы.

И с с л е д о а н и е $\Omega_{1}$. Положим ради краткости

$$
\begin{aligned}
& \xi_{i j}:=\sup _{\alpha \in U(a), \beta \in V(b)} \mid s\left[\alpha^{\top}\left(\mathbf{X}_{i}-\mathbf{X}_{j}\right) \beta^{\top}\left(\mathbf{Y}_{i}-\mathbf{Y}_{j}\right)\right] \\
&-s\left[a^{\top}\left(\mathbf{X}_{i}-\mathbf{X}_{j}\right) b^{\top}\left(\mathbf{Y}_{i}-\mathbf{Y}_{j}\right)\right] \mid \\
& \gamma_{i j}:= \sup _{\alpha \in U(a)}\left|s\left[\alpha^{\top}\left(\mathbf{X}_{i}-\mathbf{X}_{j}\right)\right]-s\left[a^{\top}\left(\mathbf{X}_{i}-\mathbf{X}_{j}\right)\right]\right| \\
& \delta_{i j}:=\sup _{\beta \in V(a)}\left|s\left[\beta^{\top}\left(\mathbf{Y}_{i}-\mathbf{Y}_{j}\right)\right]-s\left[b^{\top}\left(\mathbf{Y}_{i}-\mathbf{Y}_{j}\right)\right]\right|
\end{aligned}
$$

Так как $|s[x]| \leqslant 1$, то $\xi_{i j} \leqslant \gamma_{i j}+\delta_{i j}$, и потому

$$
\Omega_{1} \leqslant \sigma_{n}^{-1} \sum_{i \neq j}\left(\gamma_{i j}+\delta_{i j}\right)
$$


Следовательно,

$$
\begin{aligned}
& \mathbf{E}\left[\Omega_{1}\right]^{2 d} \leqslant \sigma_{n}^{-2 d} \sum_{i_{1} \neq j_{1}} \sum_{i_{2} \neq j_{2}} \cdots \sum_{i_{2 d} \neq j_{2 d}} \mathbf{E}\left(\gamma_{i_{1} j_{1}}+\delta_{i_{1} j_{1}}\right) \\
& \times\left(\gamma_{i_{2 j} j_{2}}+\delta_{i_{2} j_{2}}\right) \times \cdots \times\left(\gamma_{i_{2 d} j_{2 d}}+\delta_{i_{2 d} j_{2 d}}\right) .
\end{aligned}
$$

Мы скажем, что сомножители $\gamma_{i_{k} j_{k}}+\delta_{i_{k} j_{k}}$ и $\gamma_{i_{l j} j_{l}}+\delta_{i_{l} j_{l}}$ связаны, если у этих сомножителей есть совпадающие значения индексов: $i_{k}=i_{l}$ или $j_{k}=j_{l}$. Два сомножителя также будем называть связанными, если они входят в цепочку последовательно связанных сомножителей. Связанные сомножители образуют группу, которую мы будем называть связкой. Сумму в правой части (28) можно представить в виде $\Sigma_{1}+\cdots+\Sigma_{2 d}$, где $\Sigma_{k}$ обозначает сумму, составленную из произведений с ровно $k$ различными связками. Например, $\Sigma_{2 d}$ объединяет все произведения, в которых связаны все сомножители. Поскольку сомножители с несовпадающими индексами независимы, каждое слагаемое в $\Sigma_{k}$ равно произведению $k$ математических ожиданий, каждое из которых относится к отдельной связке. По лемме каждое из этих математических ожиданий может быть ограничено сверху:

$$
\mathbf{E}\left(\gamma_{i_{k} j_{k}}+\delta_{i_{k} j_{k}}\right) \leqslant \text { const } \cdot h .
$$

Здесь $\gamma_{i_{k} j_{k}}+\delta_{i_{k} j_{k}}$ есть элемент из связки, а постоянная зависит только от $d$ и $C$ из леммы. Число слагаемых в $\Sigma_{k}$ не превосходит $n^{2 d} n^{k}$, и потому $\Sigma_{k} \leqslant$ const $\cdot n^{2 d+k} h^{k}$, так что

$$
\mathbf{E}\left[\Omega_{1}\right]^{2 d} \leqslant \text { const } \cdot \sigma_{n}^{-2 d}\left(n^{4 d} h^{2 d}+\cdots+n^{2 d+1} h\right) .
$$

Предположим теперь, что $n h \rightarrow \infty$ и $\sqrt{n} h \rightarrow 0$ при $n \rightarrow \infty$. Тогда

$$
\mathbf{E}\left[\Omega_{1}\right]^{2 d} \leqslant \text { const } \cdot n^{4 d} h^{2 d} \sigma_{n}^{-2 d} \leqslant \text { const } \cdot h^{2 d} n^{d},
$$

если $d>p+q-2$. Тем самым, (21) доказано для $\Omega_{1}$.

И с сле дов а н и е $\Omega_{2}$. Рассмотрим

$$
\mathbf{E}\left[\Omega_{2}\right]^{2 d}=\sigma_{n}^{-2 d}\left|\sum_{i_{1} \neq j_{1}} \sum_{i_{2} \neq j_{2}} \cdots \sum_{i_{2 d} \neq j_{2 d}} \mathbf{E}\left(\phi_{i_{1} j_{1}} \phi_{i_{2} j_{2}} \cdots \phi_{i_{2 d} j_{2 d}}\right)\right|,
$$

где

$\phi_{i j}=s\left[\boldsymbol{a}^{\top}\left(\mathbf{X}_{i}-\mathbf{X}_{j}\right) b^{\top}\left(\mathbf{Y}_{i}-\mathbf{Y}_{j}\right)\right]-A\left(a, \mathbf{X}_{i}\right) B\left(b, \mathbf{Y}_{i}\right)-A\left(a, \mathbf{X}_{j}\right) B\left(b, \mathbf{Y}_{j}\right)$.

Используя введенное выше разделение на связки, легко убедиться, что для каждого $k=2 d, \ldots, d+1$ сумма $\Sigma_{k}$ содержит сомножитель, не связанный с остальными. Поскольку $\mathbf{E} \phi_{i j}=0$, для этих $k$ мы получаем, что $\mathbf{E} \Sigma_{k}=0$. Далее, поскольку $\phi_{i j}$ суть члены разложения Хёфдинга 
для $U$-статистики

$$
\left(\begin{array}{l}
n \\
2
\end{array}\right)^{-1} \sum_{1 \leqslant i<j \leqslant n} s\left[a^{\top}\left(\mathbf{X}_{i}-\mathbf{X}_{j}\right) b^{\top}\left(\mathbf{Y}_{i}-\mathbf{Y}_{j}\right)\right]
$$

они попарно ортогональны, $[1$, c. 148-149]. Далее, для каждого $k, d \geqslant$ $k \geqslant \frac{2}{3} d+1$, сумма $\Sigma_{k}$ содержит пару сомножителей, не связанную с остальными, и потому $\mathbf{E} \Sigma_{k}=0$ также и для этих $k$. Таким образом, число ненулевых слагаемых в (30) не превосходит $n^{2 d+2 d / 3+1}+\cdots+n^{2 d}$, и потому

$$
\mathrm{E}\left[\Omega_{2}\right]^{2 d} \leqslant \text { const } \cdot \sigma_{n}^{-2 d} n^{2 d+2 d / 3+1} \leqslant \text { const } \cdot n^{-d / 3+1}
$$

Предположив, что $\sqrt{n} h \rightarrow 0$ при $n \rightarrow \infty$, и взяв $d>\frac{3}{2}(p+q)$, мы получим (21) для $\Omega_{2}$.

И с с л е д о в н и е $\Omega_{3}$. Из неравенств (22) следует неравенство:

$$
\begin{aligned}
& \left|A\left(\alpha^{\prime}, \mathbf{X}\right) B\left(\beta^{\prime}, \mathbf{Y}\right)-A\left(\alpha^{\prime \prime}, \mathbf{X}\right) B\left(\beta^{\prime \prime}, \mathbf{Y}\right)\right| \\
& \quad \leqslant 2 C\left(\left|\alpha^{\prime}-\alpha^{\prime \prime}\right|+\left|\beta^{\prime}-\beta^{\prime \prime}\right|\right) .
\end{aligned}
$$

Отсюда следует, что

$$
\sup _{\alpha \in U(a), \beta \in V(b)} \sum_{i=1}^{n}\left|A\left(\alpha, \mathbf{X}_{i}\right) B\left(\beta, \mathbf{Y}_{i}\right)-A\left(a, \mathbf{X}_{i}\right) B\left(b, \mathbf{Y}_{i}\right)\right| \leqslant \text { const } \cdot n h
$$

и что $\Omega_{3}<\left(2 C_{1} n^{2} / \sigma_{n}\right) h \leqslant C_{1} \sqrt{n} h$. Поэтому $\mathbf{E}\left[\Omega_{3}\right]^{2 d} \leqslant$ const $\times$ $(\sqrt{n} h)^{2 d}$. Предположим теперь, что $\sqrt{n} h \rightarrow 0$ при $n \rightarrow \infty$ и что $d>2(p+q-2)+1$. При этих предположениях получаем, что $h^{-(p+q-2)}(\sqrt{n} h)^{2 d} \longrightarrow 0$ при $n \rightarrow \infty$. Следовательно, (21) выполнено для $\Omega_{3}$. Соединяя полученные неравенства для $\Omega_{i}, i=1,2,3$, мы видим, что (21) выполняется, если положить $h=n^{-3 / 4}$ и $d>2(p+q)-1$. Это завершает доказательство утверждения (i).

Доказательство утверждения (ii). Сходимость конечномерных распределений следует из центральной предельной теоремы для одинаково распределенных слагаемых. Полнота соответствующего семейства мер в пространстве непрерывных функций, заданных на прямом произведении сфер, следует из неравенства

$$
\begin{aligned}
& \mathbf{E}\left[A\left(\alpha^{\prime}, \mathbf{X}\right) B\left(\beta^{\prime}, \mathbf{Y}\right)-A\left(\alpha^{\prime \prime}, \mathbf{X}\right) B\left(\beta^{\prime \prime}, \mathbf{Y}\right)\right]^{2} \\
& \quad \leqslant \text { const }\left(\left|\alpha^{\prime}-\alpha^{\prime \prime}\right|^{2}+\left|\beta^{\prime}-\beta^{\prime \prime}\right|^{2}\right)
\end{aligned}
$$

В свою очередь это неравенство есть следствие (32). Теорема 1 доказана. 
4. Доказательство теоремы 2. Теорема 2 вытекает из соответствующего общего результата для гауссовских полей на гладких многообразиях: следствие 7.2 из [6]. Впервые этот результат был доказан в [5]. Исследования в [5] были вызваны нашими предшествуюшими исследованиями по непараметрической статистике [7]. Цитируем упомянутое следствие 7.2:

Теорема 3. Пусть $X(\mathbf{t})$ - гауссовское случайное поле с нулевым средним и постоянной едининной дисперсией, дифферениируемое в среднем квадратическом, которое определено на некоторой области $U \subset \mathbf{R}^{n}$. Тогда для всякого $r \leqslant n$ и всякого гладкого компакта $\mathscr{M}^{r} \subset U$ размерности $r \leqslant n$ такого, что

$$
\mathbf{E} X(\mathbf{t}) X(\mathbf{s})<1
$$

для всех $\mathbf{t}, \mathbf{s} \in \mathscr{M}^{r}, \mathbf{t} \neq \mathbf{s}$, имеет место асимптотическое представленue:

$$
\mathbf{P}\left\{\max _{\mathbf{t} \in \mathscr{M}^{r}} X(\mathbf{t})>u\right\}=\frac{1}{(\sqrt{2 \pi})^{r+1}} \int_{\mathscr{M}^{r}}\left|\operatorname{det} D_{\mathbf{s}}\right|^{1 / 2} d \mathbf{s} u^{r-1} e^{-u^{2} / 2}(1+o(1))
$$

при $u \rightarrow \infty$. Здесь $D_{\mathbf{s}}$ обозначает матричу ковариаций проекчии градиента поля $X(\mathrm{t})$ на касательное пространство многообразия $\mathscr{M}^{r}{ }_{6}$ morke s.

Вычислим определитель матрицы $D_{\mathbf{s}}$ для гауссовского поля, описанного теоремой 1 , считая $\mathbf{s}=(\alpha, \beta)$. Пусть $B_{(\alpha, \beta)}$ обозначает матрицу ковариаций градиента поля $Z(\alpha, \beta)$ в точке $(\alpha, \beta) \in S^{p-1} \times S^{q-1}$. Рассмотрим ортогональную систему координат в $\mathbf{R}^{p+q}$ с началом в точке $(\alpha, \beta)$, два из ортов которой суть $\left(\alpha, \mathbf{0}_{q}\right),\left(\mathbf{0}_{p}, \beta\right)$, где $\mathbf{0}_{p}$ и $\mathbf{0}_{q}$ обозначают нулевые векторы в $\mathbf{R}^{p}$ и $\mathbf{R}^{q}$. Остальные орты при этом лежат в касательном $(p+q-2)$-мерном линейном пространстве $T_{(\alpha, \beta)}$. Так как поле $Z$ постоянно вдоль любой четверть-плоскости $\{(t \alpha, s \beta), t \geqslant 0$, $s \geqslant 0\}$, матрица ковариаций случайного вектора $\operatorname{grad} Z(\alpha, \beta)$ в описанной координатной системе равна

$$
\widetilde{B}_{(\alpha, \beta)}=\left(\begin{array}{cccc}
0 & 0 & \cdots & 0 \\
0 & 0 & \cdots & 0 \\
\vdots & \vdots & D_{(\alpha, \beta)} \\
0 & 0 &
\end{array}\right)=Q^{\top} B_{(\alpha, \beta)} Q
$$

где $Q$ - это ортогональная матрица перехода к новой системе координат. Очевидно, что $\operatorname{det} D_{(\alpha, \beta)}$ равен сумме главных миноров порядка $p+q-2$ матрицы $\widetilde{B}_{(\alpha, \beta)}$. Эта сумма главных миноров, которую обозначим через $\tilde{\delta}\left(\tilde{B}_{(\alpha, \beta)}\right)$, инвариантна относительно выбора ортогональной системы координат. Следовательно, $\operatorname{det} D_{(\alpha, \beta)}=\tilde{\delta}\left(B_{(\alpha, \beta)}\right)$. 
Теперь вычислим $\tilde{\delta}\left(B_{(\alpha, \beta)}\right)$. Корреляционная функция поля $Z$ равна произведению корреляционных функций $r_{\mathbf{X}}\left(\alpha, \alpha^{\prime}\right)$ и $r_{\mathbf{Y}}\left(\beta, \beta^{\prime}\right)$, и потому

$$
B_{(\alpha, \beta)}=\operatorname{diag}\left[B_{\alpha}, B_{\beta}\right],
$$

где $B_{\alpha}$ и $B_{\beta}$ суть матрицы ковариаций первых $p$ координат и последних $q$ координат градиента $Z$. Поскольку поле $Z$ постоянно вдоль каждой четверть-плоскости $\{(t \alpha, s \beta), t \geqslant 0, s \geqslant 0\}$, обе матрицы $B_{\alpha}$ и $B_{\beta}$ вырождены. Следовательно, в нуль обращаются миноры, содержащие определитель матрицы $B_{\alpha}$ либо $B_{\beta}$. Поэтому сумма главных миноров порядка $p+q-2$ матрицы $B_{(\alpha, \beta)}$ равна произведению: сумма главных миноров матрицы $B_{\alpha}$ порядка $p-1$ умножается на сумму главных миноров матрицы $B_{\beta}$ порядка $q-1$ :

$$
\tilde{\delta}\left(B_{(\alpha, \beta)}\right)=\delta\left(B_{\alpha}\right) \delta\left(B_{\beta}\right) .
$$

Вычислим матрицу $B_{\alpha}$. Матрица $B_{\beta}$ вычисляется аналогично. Так как

$$
\mathbf{E} s\left[\alpha^{\prime \top}\left(\mathbf{X}_{1}-\mathbf{X}_{3}\right) \alpha^{\prime \prime \top}\left(\mathbf{X}_{2}-\mathbf{X}_{3}\right)\right]=\mathbf{E} A\left(\alpha^{\prime}, \mathbf{X}_{3}\right) A\left(\alpha^{\prime \prime}, \mathbf{X}_{3}\right),
$$

то корреляционная функция, соответствуюшая $B_{\alpha}$, равна

$$
r_{\mathbf{X}}\left(\alpha^{\prime}, \alpha^{\prime \prime}\right)=1-\frac{3}{2} \mathbf{E}\left[A\left(\alpha^{\prime}, \mathbf{X}\right)-A\left(\alpha^{\prime \prime}, \mathbf{X}\right)\right]^{2} .
$$

Для вычисления $B_{\alpha}$ достаточно вычислить асимптотику интеграла

$$
\mathbf{E}\left[A\left(\alpha^{\prime}, \mathbf{X}\right)-A\left(\alpha^{\prime \prime}, \mathbf{X}\right)\right]^{2}=\int_{\mathbf{R}^{p}}\left[A\left(\alpha^{\prime}, \mathbf{x}\right)-A\left(\alpha^{\prime \prime}, \mathbf{x}\right)\right]^{2} p_{\mathbf{X}}(\mathbf{x}) d \mathbf{x}
$$

при $\alpha^{\prime}-\alpha^{\prime \prime} \rightarrow$ 0. Используя (23), (24), по формуле Тейлора получаем

$$
\begin{gathered}
\mathbf{P}\left\{\alpha^{\prime \top} \mathbf{X}<\alpha^{\prime \top} \mathbf{x}\right\}-\mathbf{P}\left\{\alpha^{\prime \prime \top} \mathbf{X}<\alpha^{\prime \prime} \mathbf{x}\right\} \\
=\delta f^{\prime}(0, \mathbf{x})+\delta\left[f^{\prime}(\theta \delta, \mathbf{x})-f^{\prime}(0, \mathbf{x})\right]
\end{gathered}
$$

для некоторого $\theta, 0 \leqslant \theta \leqslant 1$. Далее, из (36) следует, что

$$
\begin{aligned}
\mathbf{E}[ & \left.A\left(\alpha^{\prime}, \mathbf{X}\right)-A\left(\alpha^{\prime \prime}, \mathbf{X}\right)\right]^{2} \\
= & 4 \int_{\mathbf{R}^{p}}\left[\mathbf{P}\left\{\alpha^{\prime \top} \mathbf{X}<\alpha^{\prime \top} \mathbf{x}\right\}-\mathbf{P}\left\{\alpha^{\prime \prime \top} \mathbf{X}<\alpha^{\prime \prime \top} \mathbf{x}\right\}\right] p_{\mathbf{X}}(\mathbf{x}) d \mathbf{x} \\
= & 4 \delta^{2} \int_{\mathbf{R}^{p}}\left[f^{\prime}(0, \mathbf{x})\right]^{2} p_{\mathbf{X}}(\mathbf{x}) d \mathbf{x} \\
& +8 \delta^{2} \int_{\mathbf{R}^{p}} f^{\prime}(0, \mathbf{x})\left[f^{\prime}(\theta \delta, \mathbf{x})-f^{\prime}(0, \mathbf{x})\right] p_{\mathbf{X}}(\mathbf{x}) d \mathbf{x} \\
& +4 \delta^{2} \int_{\mathbf{R}^{p}}\left[f^{\prime}(\theta \delta, \mathbf{x})-f^{\prime}(0, \mathbf{x})\right]^{2} p_{\mathbf{X}}(\mathbf{x}) d \mathbf{x} .
\end{aligned}
$$

Для любого $\mathbf{x}$ подынтегральные выражения в двух последних интегралах стремятся к 0 , когда $\delta \rightarrow 0$. Поэтому и сами интегралы стремятся 
к 0 по теореме о мажорированной сходимости, так как $f^{\prime}(\delta, \mathbf{x})$ равномерно ограничена. Поэтому два последних слагаемых суть $O\left(\delta^{2}\right)$ при $\delta \rightarrow 0$. В силу (26) первое слагаемое можно выразить через исходные переменные. Приняв во внимание (35), получим, что

$$
\begin{aligned}
r_{\mathbf{X}}\left(\alpha^{\prime}, \alpha^{\prime \prime}\right)= & 1-6\left(\alpha^{\prime}-\alpha^{\prime \prime}\right)^{\top} \\
& \times\left[\int_{\alpha^{\prime}{ }^{\top} \mathbf{x}=0} \int_{\alpha^{\prime \prime}{ }^{\top} \mathbf{y}=0} \mathbf{x y}^{\top} d \mathbf{x} d \mathbf{y}\right. \\
& \left.\times \int p_{\mathbf{X}}(\mathbf{x}+\mathbf{z}) p_{\mathbf{X}}(\mathbf{y}+\mathbf{z}) p_{\mathbf{X}}(\mathbf{z}) d \mathbf{z}\right] \\
& \times\left(\alpha^{\prime}-\alpha^{\prime \prime}\right)+o\left(\left|\alpha^{\prime}-\alpha^{\prime \prime}\right|^{2}\right) .
\end{aligned}
$$

Это дает нам выражение для $B_{\alpha}$, так что $B_{\alpha}=B_{\mathbf{X}}(\alpha)$, см. (12). Теперь обратимся к условию невырожденности (33). Заметим, что в силу (7) и слабой сходимости (ii) для всех $\alpha \in \mathbf{R}^{p}, \beta \in \mathbf{R}^{q}$ справедливо равенство $Z(\alpha, \beta)=Z(-\alpha,-\beta)$, откуда

$$
r(\alpha, \beta ;-\alpha,-\beta)=1 .
$$

Поэтому теорему 3 нельзя применить непосредственно. Наша цель сейчас - выделить такой компакт $T \subset S^{p-1} \times S^{q-1}$, для которого справедливо

$$
\sup _{T} Z(\alpha, \beta)=\sup _{|\alpha|=|\beta|=1} Z(\alpha, \beta)
$$

и для любых точек $\mathbf{t}, \mathbf{s}, \mathbf{t} \neq \mathbf{s}$, которого выполняется (33).

Допустим, что для некоторьх $\left(\alpha^{\prime}, \beta^{\prime}\right) \neq\left(\alpha^{\prime \prime}, \beta^{\prime \prime}\right)$, тем не менее,

$$
r\left(\alpha^{\prime}, \beta^{\prime} ; \alpha^{\prime \prime}, \beta^{\prime \prime}\right)=1 \text {. }
$$

Тогда либо $r_{\mathbf{X}}\left(\alpha^{\prime}, \alpha^{\prime \prime}\right)=1$, либо $r_{\mathbf{X}}\left(\alpha^{\prime}, \alpha^{\prime \prime}\right)=-1$. Предположим, что верно первое. В силу (23) и (35)

$$
\mathbf{P}\left\{\alpha^{\prime \top} \mathbf{X}<\alpha^{\prime \top} \mathbf{x}\right\}=\mathbf{P}\left\{\alpha^{\prime \prime \top} \mathbf{X}<\alpha^{\prime \prime \top} \mathbf{x}\right\}
$$

для почти всех $\mathbf{x}$ в смысле исходной вероятностной меры. Пусть теперь $\mathbf{x} \in \mathbf{R}^{p}$ пробегает гиперплоскость $\alpha^{\prime \top} \mathbf{x}=$ const. При этом левая часть (42) остается неизменной. Поскольку $\alpha^{\prime \prime \top} \times$ и, следовательно, правая часть (42) при этом не остаются постоянными, высказанное выше предположение приводит нас к противоречию.

Покажем теперь, что $r_{\mathbf{X}}\left(\alpha, \alpha^{\prime}\right)=-1$, если и только если $\alpha=-\alpha^{\prime}$. В самом деле, в силу (7) и слабой сходимости (ii) из первого равенства следует, что $r_{\mathbf{X}}\left(\alpha,-\alpha^{\prime}\right)=1$, и потому, по доказанному выше, $\alpha=-\alpha^{\prime}$. Обратное утверждение очевидно.

Пусть $\alpha_{1}$ обозначает первую координату вектора $\alpha \in S^{p-1}$. Положим

$$
T=\left\{\alpha: \alpha_{1}>0\right\} \cap\left\{S^{p-1} \times S^{q-1}\right\} .
$$


В силу (39) условие (40) для $T$ выполняется. В то же время, из сказанного выше следует, что если для некоторых $\left(\alpha^{\prime}, \beta^{\prime}\right) \in T$ и $\left(\alpha^{\prime \prime}, \beta^{\prime \prime}\right) \in T$ выполняется (41), то $\left(\alpha^{\prime}, \beta^{\prime}\right)=\left(\alpha^{\prime \prime}, \beta^{\prime \prime}\right)$. Поэтому условие (7) справедливо для $T$. Для окончания доказательства теоремы остается заметить, что

$$
\begin{aligned}
\int_{T} & \sqrt{\delta\left(B_{\mathbf{X}}(\alpha)\right) \delta\left(B_{\mathbf{Y}}(\beta)\right)} S(d \alpha) S(d \beta) \\
& =\frac{1}{2} \int_{S^{p-1} \times S^{q-1}} \sqrt{\delta\left(B_{\mathbf{X}}(\alpha)\right) \delta\left(B_{\mathbf{Y}}(\beta)\right)} S(d \alpha) S(d \beta)=\frac{1}{2} K_{\mathbf{X}} K_{\mathbf{Y}}
\end{aligned}
$$

5. Оценивание $K_{\mathbf{X}}$ и $K_{\mathbf{Y}}$. В этом пункте мы представляем состоятельную оценку для $K_{\mathbf{X}} K_{\mathbf{Y}}$, которой можно заместить константу $K_{\mathbf{X}} K_{\mathbf{Y}}$ при применении предложенного критерия. В несколько иных обстоятельствах оценки для $K_{\mathbf{X}}$ и $K_{\mathbf{Y}}$ уже обсуждались в [7]. Ниже мы описываем правило оценивания $K_{\mathbf{X}}$ по выборке $\mathbf{X}_{1}, \ldots, \mathbf{X}_{n}$. Положим

$$
F_{i}(\alpha, \mathbf{x})=\int_{\alpha^{\top} \mathbf{y}=0} y_{i} p_{\mathbf{X}}(\mathbf{x}-\mathbf{y}) d \mathbf{y}
$$

Элемент $b_{\alpha}(i, j)$ матрицы $B_{\mathbf{X}}(\alpha)$ имеет вид

$$
b_{\alpha}(i, j)=\int F_{i}(\alpha, \mathbf{x}) F_{j}(\alpha, \mathbf{x}) p_{\mathbf{X}}(\mathbf{x}) d \mathbf{x} .
$$

Чтобы построить оценку для $K_{\mathbf{X}}$, приблизим $F_{i}(\alpha, \mathbf{x})$ посредством

$$
F_{i}^{(h)}(\alpha, \mathbf{x})=\frac{1}{h} \int_{-h / 2 \leqslant \alpha^{\top} \mathbf{y} \leqslant h / 2} y_{i} p_{\mathbf{X}}(\mathbf{x}-\mathbf{y}) d \mathbf{y},
$$

где $h>0-$ малое число. Величину $F_{i}^{(h)}(\alpha, \mathbf{x})$ можно заменить ее оценкой, получаемой усреднением по выборке. Пусть $\mathbf{X}_{i}=\left(X_{i 1}, \ldots, X_{i p}\right)$, $i=1, \ldots, n$. Используя эту координатную форму наблюдений, введем матрицу $B_{\alpha}^{n}$ размера $p \times p$, задав ее элементы следующей формулой:

$$
\begin{aligned}
& b_{\alpha}^{n}(i, j)= \frac{12}{n^{3} h^{2}} \sum_{m=1}^{n} \sum_{k=1}^{n} \sum_{l=1}^{n}\left(X_{m i}-X_{k i}\right)\left(X_{m j}-X_{l j}\right) \\
& \times \mathbf{I}_{\alpha^{\top}} \mathbf{X}_{m}-h / 2 \leqslant \alpha^{\top} \mathbf{X}_{k} \leqslant \alpha^{\top} \mathbf{X}_{m}+h / 2 \\
& \mathbf{I}_{\alpha} \mathbf{X}_{m}-h / 2 \leqslant \alpha^{\top} \mathbf{X}_{l \leqslant \alpha^{\top}} \mathbf{X}_{m}+h / 2
\end{aligned}
$$

$i, j=1, \ldots, p$.

Теорема 4. Пусть функиии

$$
\begin{array}{ll}
G_{i j k l}(\alpha, \mathbf{x})=\int_{\alpha^{\top} \mathbf{z}=0} z_{i} z_{j} z_{k} z_{l} p_{\mathbf{X}}(\mathbf{x}-\mathbf{z}) d \mathbf{z}, & \mathbf{x} \in \mathbf{R}^{p}, \\
G_{i j k l}(\beta, \mathbf{y})=\int_{\beta^{\top} \mathbf{z}=0} z_{i} z_{j} z_{k} z_{l} p_{\mathbf{Y}}(\mathbf{y}-\mathbf{z}) d \mathbf{z}, & \mathbf{y} \in \mathbf{R}^{q},
\end{array}
$$


равномерно ограничены по $\alpha$ u $\mathbf{x}$ для всех $i, j, k, l=1, \ldots, p$ и по $\beta$ и $\mathbf{y}$ для всех $i, j, k, l=1, \ldots, q$. Тогда

$$
K_{\mathbf{X}}^{n}=\int_{S^{p-1}}\left|\delta\left(B_{\alpha}^{n}\right)\right|^{1 / 2} S(d \alpha) \quad u \quad K_{\mathbf{Y}}^{n}=\int_{S^{q-1}}\left|\delta\left(B_{\beta}^{n}\right)\right|^{1 / 2} S(d \beta)
$$

по вероятности сходятся $\kappa K_{\mathbf{X}} u K_{\mathbf{Y}}$ соответственно, если $h=h_{n}$ въцбано так, ито $h_{n} \rightarrow 0, n h_{n} \rightarrow \infty$ nрu $n \rightarrow \infty$.

Доказательство этой теоремы опирается на простейшие свойства интегралов и сводится к прямым вычислениям математических ожиданий и дисперсий для величин $b_{\alpha}^{n}(i, j), i, j=1, \ldots, p$, и $b_{\beta}^{n}(i, j), i, j=$ $1, \ldots, q$. Поэтому мы его не приводим.

Авторы признательны анонимному рецензенту и Т. А. Романовой за внимание к работе и полезные замечания.

\section{СПИСОК ЛИТЕРАТУРЫ}

1. Ghosh M. Rank statistics and limit theorems. - Nonparametric Methods. Handbook of Statistics, v. 4. Ed. by K. Krishnaiah and P. K. Sen. Amsterdam: North-Holland, 1984, p. $145-171$.

2. Гихман И. И., Скороход А. В. Введение в теорию случайных процессов. М.: Наука, 1965, $656 \mathrm{c.}$

3. Kendall M., Gibbons J.D. Rank Correlation Methods. New York: Oxford Univ. Press, 1990, $260 \mathrm{p}$.

4. Леман Э. Проверка статистических гипотез. М.: Наука, 1964, 498 с.

5. Михалева Т.Л., Питербара В.И. О распределении максимума гауссовского поля с постоянной дисперсией на гладком многообразии. - Теория вероятн. и ее примен., 1996, т. 41, в. 2, с. 438-451.

6. Piterbarg V.I. Asymptotic Methods in the Theory of Gaussian Processes and Fields. Providence, RI: Amer. Math. Soc., 1996, 206 p.

7. Piterbarg V., Tyurin Yu. Testing for homogeneity of two multivariate samples: a Gaussian field on a sphere. - Math. Methods Statist., 1993, v. 2, p. 147-164.

8. Puri M.L., Sen P.K. Nonparametric Methods in Multivariate Analysis. New York: Wiley, 1971, $440 \mathrm{p}$.

9. Roy S.N. On a heuristic method of test construction and its use in multivariate analysis. - Ann. Math. Statist., 1953, v. 24, p. 220-238.

10. Roy S. N. Some Aspects of Multivariate Analysis. New York: Wiley, 1957.

11. Крамер Г. Математические методы статистики. М.: Мир, 1975, 648 с.

12. Андерсон Т. Введение в многомерный статистический анализ. М.: Физматгиз, $1963,500 \mathrm{c}$.

13. Гаек Я., ШІидах 3. Теория ранговых критериев. М.: Наука, 1971, 376 с.

14. Холлендер М., Вул $\phi$ Д. Непараметрические методы статистики. М.: Финансы и статистика, 1983,518 с.

15. Mötonen J., Oja $H$. Multivariate spatial sign and rank methods. - Nonparametr. Statist., 1995, v. 5, № 2, p. 201-213.

16. Randles $R$. H., Peters $D$. Multivariate rank tests for the two-sample location problem. - Comm. Statist. Theory Methods, 1986, v. 15, № 11, p. 4225-4238. 